Europhys. Lett., 45 (2), pp. 222-227 (1999)

\title{
Interdependence of strain and shape in self-assembled coherent InAs islands on GaAs
}

\author{
I. Kegel ${ }^{1}$, T. H. Metzger ${ }^{1}$, P. Fratzl $\left.{ }^{1}{ }^{*}\right)$, J. Peisl ${ }^{1}$, A. Lorke $^{1}$ \\ J. M. Garcia ${ }^{2}$ and P. M. PetrofF ${ }^{2}$ \\ 1 Sektion Physik, Ludwig-Maximilians-Universität München \\ D-80539 München, Germany \\ ${ }^{2}$ Materials Department, University of California \\ Santa Barbara, CA 93106, USA
}

(received 13 July 1998; accepted in final form 16 November 1998)

PACS. 68.65+g - Low-dimensional structures (superlattices, quantum well structures, multilayers): structure, and nonelectronic properties.

PACS. 68.55Jk - Structure and morphology; thickness.

PACS. 61.10-i - X-ray diffraction and scattering.

\begin{abstract}
Self-assembled coherent InAs islands on GaAs (100) have been investigated by a novel version of grazing-incidence diffraction ("iso-strain scattering"). This method permits the determination of the interdependence of strain and shape, as well as the relaxation gradient within the InAs dots. The relaxation in the islands ranges from fully strained at the bottom to completely relaxed at the top of the islands. The radius of the dots at a given height depends linearly on the local elastic lattice relaxation, with a rapidly increasing relaxation gradient when approaching the top of the islands.
\end{abstract}

A large number of potential device applications for dislocation-free quantum dots have been proposed in the past few years [1]. Since millions of quantum dots are needed in order to yield macroscopic effects, a narrow-size distribution is crucial for adequate performance. Dislocation-free growth and narrow-size distribution are obtained intrinsically during StranskiKrastanow growth in strained layer heteroepitaxy. A recent study [2] indicates that total energy calculations $[3,4]$ alone are not sufficient to explain the origin and structure of selfassembled islands grown in the Stranski-Krastanow mode. Formation of quantum dots is sensitively dependent on growth rates and temperatures, rather than just on total monolayer coverage. Dobbs et al. [2] emphasise that the islands are the metastable final stage of a complicated kinetic process. The validity of kinetic rate equations has to be tested against experimental data on the final structure of the islands. The shape of the surface dots is accessible by atomic force microscopy (AFM) measurements, from which shape and spatial distribution can be determined [5]. A more profound insight into the actual process requires

$\left({ }^{*}\right)$ Permanent address: Erich Schmid Institute of Solid State Physics, Austrian Academy of Sciences, University of Leoben, A-8700 Leoben, Austria.

(C) EDP Sciences 
knowledge about the strain distribution inside the islands. Strain, as the driving force for the Stranski-Krastanow growth, also influences the electronic properties of the dots. Finite element calculations show that elastic strain relaxation in unburied dots $[6,7]$ ranges from fully strained at the bottom to completely relaxed at the top of the islands. Together with shape (confinement), it determines band gap and quantisation of electron levels [6,8]. Strain can most easily be measured with diffraction experiments. So far, information has mainly been obtained from conventional high-resolution large-angle X-ray diffraction $[9,10]$ on islands embedded in superlattices. There, the lack of depth resolution and the dominating influence of the substrate severely limit the separation of shape and strain effects. Only the average strain was derived and surface dot effects could not be distinguished from the contribution of buried islands. In case of faceted Ge pyramids on $\mathrm{Si}$, the corresponding crystal truncation rods have been used to obtain detailed information on the strain inside the islands [11]. In the present InAs/GaAs system this method is not applicable since the InAs island perimeter is not faceted.

In this letter we demonstrate that X-ray grazing-incidence diffraction permits the investigation of the interdependence of shape and strain in islands. This technique yields depthsensitive structural information on very thin vertically and laterally structured semiconductor systems $[12,13]$ such as quantum wells and superlattices or quantum wires, respectively. Refraction effects, at incidence and exit angles close to the critical angle of total external reflection reduce the scattering depth to a range between $5 \mathrm{~nm}$ and $1 \mu \mathrm{m}$, thereby emphasising the scattering from near- or on-surface structures. InAs islands were grown on GaAs by MBE at $530{ }^{\circ} \mathrm{C}$ in the Stranski-Krastanow growth mode. The InAs coverage was kept low enough to avoid lateral dot correlations (island density $10^{9} / \mathrm{cm}^{2}$ ), i.e. the two-point dot correlation shows a Poisson distribution [14]. The AFM analysis reveals isotropic dot diameters of $34 \mathrm{~nm}$ and heights of $10 \mathrm{~nm}$ [15]. In the present case of uncovered coherent InAs islands grown on GaAs, we use 3D reciprocal space mapping in the vicinity of the GaAs (220) reciprocal lattice point. In a basic model, the sample is considered as a perfect GaAs single crystal covered with randomly distributed, albeit coherent InAs islands in which the lattice parameter and the outer perimeter are assumed to change as functions of the height. Hence, since the coherent Stranski-Krastanow growth mode is dislocation-free, the expected scattering is solely due to intrinsic strain- and shape-related contributions.

The grazing-incidence diffraction measurements have been performed at the beamline BW2 at the synchrotron radiation source DORIS at Hasylab, DESY, Hamburg. The reciprocal space has been mapped out by angular scans at different radial positions. The intensity distribution in the third direction perpendicular to the sample surface was simultaneously recorded by a position-sensitive detector as described earlier [12]. The wavelength was set to a relatively large value $(2.06 \AA)$ to improve on resolution at the given angular divergence of the instrument. The measurement was performed at an incidence angle $\alpha_{i}$ below the critical angle for total external reflection, which limits the penetration depth of the X-rays to about 10 $\mathrm{nm}$ only, thereby suppressing unwanted scattering from the substrate. The resolution element, as determined by the beamline optics in front of the sample and the collimation path after the sample, was small enough to justify the neglection of resolution corrections during data evaluation. The footprint of the X-rays on the sample surface was about $1 \mathrm{~mm}^{2}$ and ensured reliable sampling statistics.

Figure 1b) shows an overview of the grazing-incidence diffraction results. The strain in the islands leads to a variation of the lattice parameter and, hence, to a spreading of the intensity distribution along the radial direction $q_{\mathrm{r}}$ from the position of the GaAs (220) at $q_{\mathrm{r}}=0$, to the position of the corresponding reflection of unstrained InAs, at $q_{\mathrm{r}}=-0.225 \AA^{-1}$. The intensity at intermediate values of $q_{\mathrm{r}}$ is then due to the strained InAs islands, with the lattice 
a)

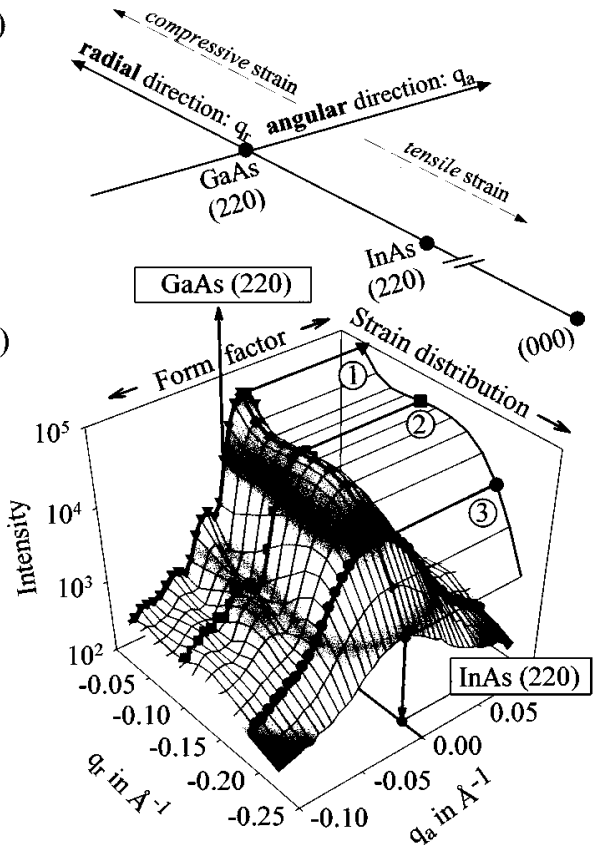

Fig. 1. - a) Schematic view of the scan directions in reciprocal space close to the (220) GaAs and InAs surface Bragg reflections. b) Dot-induced intensity distribution between the GaAs and InAs (220) surface reflections. The strain-dependent intensity change is projected onto the $I-q_{\mathrm{r}}$ plane. The dot form-factor-induced intensity variation appears along the $q_{\mathrm{a}}$-direction. The mesh represents all measured $q_{\mathrm{r}}-q_{\mathrm{a}}$ scans. From the 12 angular scans, three strain levels at $q_{\mathrm{r}}$-positions 1-3 are emphasized and show that the lateral dimension of scattering objects increases with decreasing relaxation (small $q_{\mathrm{r}}$-values).

parameter varying from the value of unstrained InAs to unstrained GaAs. If the islands were infinitely extended layers, then all the scattering intensity would be concentrated on the radial axis defined by $q_{\mathrm{a}}=0$. The finite extension of the islands, however, leads to a broadening in the $q_{\mathrm{a}^{-}}$and $q_{\mathrm{r}}$-direction. While the $q_{\mathrm{r}}$-broadening is hidden by the coherent addition of the form-factor-induced scattering amplitudes, we will show below that the $q_{\mathrm{a}}$-broadening is solely due to the form factor of the islands and thus, a separation between strain and shape effects will be possible. The meaning of radial and angular directions, $q_{\mathrm{r}}$ and $q_{\mathrm{a}}$, respectively, is explained in fig. 1a), where the reciprocal space close to the relevant (220) surface Bragg-reflections is sketched.

Three form-factor-dominated scans along $q_{\mathrm{a}}$ are emphasised in fig. $1 \mathrm{~b}$ ) and plotted in fig. 2a). The inset shows the dependence of the scattering intensity along $q_{\mathrm{r}}$ for $q_{\mathrm{a}}=0$. Qualitatively, they permit preliminary conclusions. The intensity distribution along $q_{\mathrm{a}}$ always exhibits a central broad maximum and several side maxima, which disappear for large $q_{\mathrm{r}}$ in the more relaxed regions of the InAs islands. With increasing relaxation, the central maximum broadens, indicating that the lateral dimension of the scattering objects decreases. Hence, the InAs islands appear larger in those areas where the lattice spacing is closer to GaAs, most likely at the foot of the islands. A second qualitative observation is the number of side maxima (up to three) in the form-factor-induced intensity distribution (fig. 2a)). This implies that the size distribution of the crystalline core of the islands must be extremely narrow (i.e. nearly monodisperse) such that a smearing of these side maxima is avoided. 


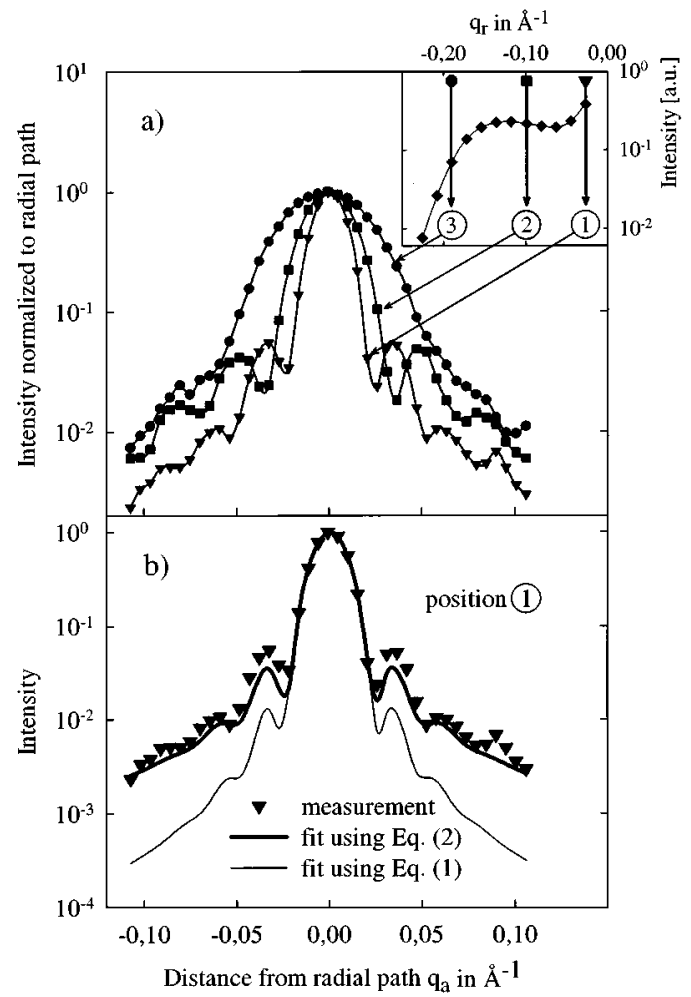

Fig. 2

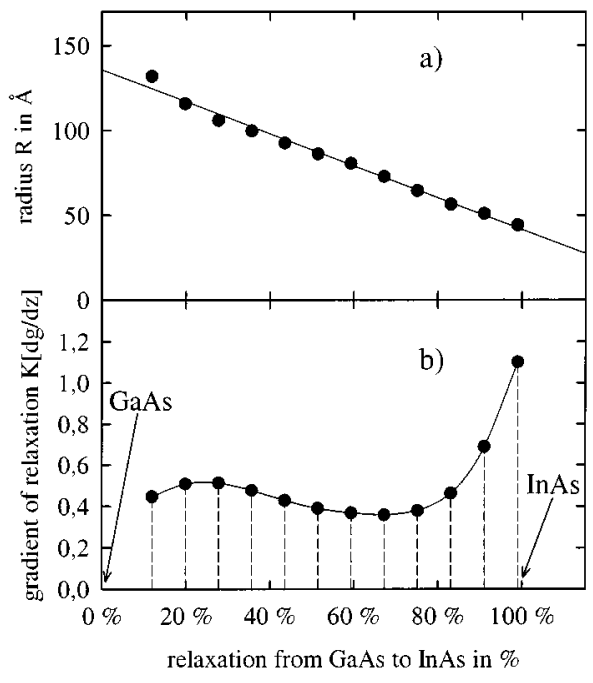

Fig. 3

Fig. 2. - a) Angular cuts along $q_{\mathrm{a}}\left(\langle 1-10\rangle\right.$ direction) at different $q_{\mathrm{r}}$-positions 1 to 3 (see inset and fig. 1). b) Comparison between model calculations and experimental data for the angular scan at position $1\left(q_{\mathrm{r}}=-0.027 \AA^{-1}\right)$ assuming the form factor for cylindrical disks (according to eq. (1), thin line) and after the integration leading to eq. (2) (thick line).

Fig. 3. - a) Dot radius $R$ and b) strain gradient $K \cdot \mathrm{d} g / \mathrm{d} z$ of the iso-strain volumes as a function of strain relaxation in percent of the relative lattice mismatch $\left(a_{\mathrm{InAs}}-a_{\mathrm{GaAs}}\right) / a_{\mathrm{GaAs}}$. The straight line in a) is a linear fit through the data. The curve in b) represents a guide to the eyes.

For an analytical description of the scattering from the islands, we use a simple model based on three assumptions: 1) The iso-strain volumes are considered to be limited by planes parallel to the substrate surface, which allows for unique identification of height and relaxation in the dot. Therefore, the reciprocal lattice parameter $g(z)$ is only a function of the height $z$ above the substrate. 2) As supported by AFM measurements [15] and our own small angle scattering results from these dots (not shown here), each dot is assumed to have full symmetry around the axis perpendicular to the substrate surface, with a radius $R(z)$ at height $z$. 3) The variation of both the radius $R(z)$ and the relaxation gradient $g^{\prime}=\mathrm{d} g / \mathrm{d} z$, is assumed to be negligibly small over a distance in reciprocal space of the order of $1 / R$.

Each "iso-strain disk" of radius $R$ scatters centro-symmetrically around its local lattice Bragg peak defined by $q_{\mathrm{r}}$ with an intensity distribution given by a first-order Bessel function:

$$
I(q)=I_{0}\left(\frac{2 J_{1}(q R)}{q R}\right)^{2} .
$$


The total scattering distribution in the angular direction is obtained from a coherent integration over iso-strain disk contributions, which, due to the strain gradient, are lined up in the radial direction $q_{\mathrm{r}}$. Together with the above-mentioned approximations, this leads to the particularly simple analytic expression

$$
I\left(q_{\mathrm{a}}\right)=I_{\max }\left(q_{\mathrm{r}}\right)\left(\frac{\sin q_{\mathrm{a}} R}{q_{\mathrm{a}} R}\right)^{2},
$$

with

$$
I_{\max }=I_{0}\left(\frac{4 \pi R}{g^{\prime}(z)}\right)^{2}
$$

Fitting the 12 angular scans and the intensity in radial direction with this model we obtain the radius of the iso-strain disks $R$ as well as the strain gradient $g^{\prime}=\mathrm{d} g / \mathrm{d} z$ as functions of the relaxation. As an example, the intensity distribution in angular direction at the $q_{\mathrm{r}}$-position 1 (see inset in figs. 2a) and 1b)) is shown in fig. 2b) together with the fits using eq. (1) (thin line) and eq. (2) (thick line), respectively. While the maximum at the centre is well reproduced by both fits, the intensity decay of the side maxima can be fitted roughly only by eq. (2). The important dot radius $R$ is, however, determined with high accuracy from the fit to the central maximum, in both models used. The scattering physics involved in a better fit of the intensity wings along $q_{\mathrm{a}}$ is currently being developed [16]. Excluding the end-of-range effects for $q_{\mathrm{r}}$ smaller than the InAs (220) position and larger than the GaAs (220) position from this data analysis, we are now able to unambiguously relate the radius $R$ to the lattice relaxation within the dot. Figure 3a) shows the interdependence of relaxation and radius $R$. The relationship is linear except for the measurement closest to the GaAs reflection, where crossover effects from the transition of the dot into the substrate can no longer be neglected. We find that the radii determined here are smaller by about $5 \mathrm{~nm}$ than the dot radius as evaluated from our small-angle scattering measurements (not shown here) or AFM studies [15]. The difference in the average radius is most likely due to the natural oxide layer which covers the "naked" InAs islands of our sample.

The strain gradient $g^{\prime}$ as a function of relaxation $\Delta a / a$ is shown in fig. $\left.3 \mathrm{~b}\right) . g^{\prime}$ follows immediately from $I_{\max }\left(q_{\mathrm{r}}\right)$, eq. (3), but remains multiplied by an unknown constant $K:=$ $4 \pi \sqrt{I_{0}}$, where $I_{0}$ is the intensity of the primary beam. Again, the first data point should be omitted from further conclusions for reasons mentioned above. The local strain gradient first decreases and then increases with the lateral relaxation and becomes strongest at the top of the dot where the InAs lattice can relax freely. Towards the bottom, the islands are squeezed by the GaAs substrate and the InAs lattice cannot relax at all. The fact that we find a Bragg peak at the position of the completely relaxed InAs lattice point (100\% relaxation in fig. 3 ) proves that at least at the top of the InAs dots no intermixing of In and Ga has taken place during growth.

We have demonstrated a new method which allows us to relate strain and shape in selfassembled coherent islands. This is important information as both quantities appreciably influence the band structure. In addition, our results will serve as a valuable tool to further understand the growth of coherent islands, since the growth mechanism in the StranskiKrastanow mode is highly strain-related. Theoretically, the interdependence of strain and shape in coherent island growth has been treated by total energy calculations $[6,7,17]$. Here, the shape of the islands is, however, artificially chosen and does not result from the growth process itself, nor can the 2D-3D transition and the narrow size distribution be explained. A new concept using kinetic arguments and microscopic rate equations [1] has been reported recently. Due to its mean-field character, spatial information is not available and a strain distribution 
remains to be incorporated into this model. Our detailed results on the interdependence of strain and shape should help to understand both growth and band structure effects of coherent islands.

We would like to acknowledge the support by the Deutsche Forschungsgemeinschaft under grant number Pe 127/1-6+2 and the help in travelling expenses by HASYLAB. We very much appreciated the technical support from the HASYLAB staff at beamline BW2. The LMU-UCSB collaboration is supported by the BMBF and a Max-Planck Forschungspreis.

\section{REFERENCES}

[1] Fafard S., Hinzer K., Raymond S., Dion M., Mccaffrey J., Feng Y. and CharbonNEAU S., Science, 274 (1996) 1350.

[2] Dobbs H. T., Vvedensky D. D., Zangwill A., Johansson J., Carlsson N. and Seifert W., Phys. Rev. Lett., 79 (1997) 897.

[3] Priester C. and Lannoo M., Phys. Rev. Lett., 75 (1995) 93.

[4] Chen Y. and Washburn J., Phys. Rev. Lett., 77 (1996) 4046.

[5] Leonard D., Pond K. and Petroff P. M., Phys. Rev. B, 50 (1994) 11689.

[6] Grundmann M., Stier O. and Bimberg D., Phys. Rev. B, 52 (1995) 11969.

[7] Yu W. and Madhukar A., Phys. Rev. Lett., 79 (1997) 905.

[8] Jiang H. and Singh J., Phys. Rev. B, 56 (1997) 4696.

[9] Darhuber A. A., Schittenhelm P., Holy V., Stangl J., Bauer G. and Abstreiter G., Phys. Rev. B, 55 (1997) 15652.

[10] Darhuber A. A. et al., Appl. Phys. Lett., 70 (1997) 955.

[11] Steinfort A. J. et al., Phys. Rev. Lett., 77 (1996) 2009.

[12] Pietsch U., Metzger H., Rugel S., Jennichen B. and Robinson I. K., J. Appl. Phys., 74 (1993) 2381.

[13] Paschke K. et al., Appl. Phys. Lett., 70 (1997) 1031.

[14] Simmel F., unpublished.

[15] Garcia J. M., Medeiros-Ribeiro G., Schmidt K., Ngo T., Feng J. L., Lorke A., Kotthaus J. and Petroff P. M., Appl. Phys. Lett., 71 (1997) 2014.

[16] Kegel I., Metzger H. and Peisl J., submitted to Phys. Rev. B.

[17] Johnson H. T. and Freund L. B., J. Appl. Phys., 81 (1997) 6081. 